\title{
Analytical Pitfalls of Therapeutic Drug Monitoring of Thiopurines in Patients With Inflammatory Bowel Disease
}

\author{
Melek Simsek, MD, * Berrie Meijer, MD, ${ }^{*}$ Chris J. J. Mulder, MD, PhD, * \\ Adriaan A. van Bodegraven, MD, PhD, *广 and Nanne K. H. de Boer, MD, PhD*
}

\begin{abstract}
The use of thiopurines in the treatment of inflammatory bowel disease (IBD) can be optimized by the application of therapeutic drug monitoring. In this procedure, 6-thioguanine nucleotides (6-TGN) and 6-methylmercaptopurine (6-MMP) metabolites are monitored and related to therapeutic response and adverse events, respectively. Therapeutic drug monitoring of thiopurines, however, is hampered by several analytical limitations resulting in an impaired translation of metabolite levels to clinical outcome in IBD. Thiopurine metabolism is cell specific and requires nucleated cells and particular enzymes for 6-TGN formation. In the current therapeutic drug monitoring, metabolite levels are assessed in erythrocytes, whereas leukocytes are considered the main target cells of these drugs. Furthermore, currently used methods do not distinguish between active nucleotides and their unwanted residual products. Last, there is a lack of a standardized laboratorial procedure for metabolite assessment regarding the substantial instability of erythrocyte 6-TGN. To improve thiopurine therapy in patients with IBD, it is necessary to understand these limitations and recognize the general misconceptions in this procedure.
\end{abstract}

Received for publication June 5, 2017; accepted August 17, 2017.

From the *Department of Gastroenterology and Hepatology, VU University Medical Center, Amsterdam; and †Department of Gastroenterology, Geriatrics, Internal and Intensive Care Medicine (Co-MIK), Zuyderland Medical Center, Heerlen-Sittard-Geleen, the Netherlands.

Guarantor of the article: N. K. H. de Boer. M. Simsek and N. K. H. de Boer conceived the study and collected all data. M. Simsek and B. Meijer screened the articles for availability. M. Simsek, B. Meijer, and N. K. H. de Boer developed the figures. M. Simsek and B. Meijer drafted the manuscript. C. J. J. Mulder, N. K. H. de Boer, and A. A. van Bodegraven critically revised the manuscript. All authors commented on drafts of the manuscript. All authors have approved the final draft of the article.

C. J. J. Mulder has served as a consultant and principal investigator for Teva Pharma B.V. A. A. van Bodegraven has served as a consultant or speaker for AbbVie, Ferring, Janssen, MSD, Pfizer, Takeda, TEVA, Tramedico, Vifor, and the Dutch Ministry of Health (ZonMW). He has received (unrestricted) research grants from Aventis and Ferring, and the Dutch Ministry of Health. N. K. H. de Boer has served as a speaker for AbbVie, Takeda, and MSD. He has served as a consultant and principal investigator for Takeda and Teva Pharma B.V. He has received a (unrestricted) research grant from Dr. Falk and Takeda. The remaining authors declare no conflict of interest.

Correspondence: Melek Simsek, MD, Department of Gastroenterology, VU University Medical Centre, room PK 2X 136, De Boelelaan 1118, 1081 HZ Amsterdam, the Netherlands (e-mail: m.simsek@vumc.nl).

Copyright $(C 2017$ The Author(s). Published by Wolters Kluwer Health, Inc. on behalf of the International Association of Therapeutic Drug Monitoring and Clinical Toxicology. This is an open-access article distributed under the terms of the Creative Commons Attribution-Non CommercialNo Derivatives License 4.0 (CCBY-NC-ND), where it is permissible to download and share the work provided it is properly cited. The work cannot be changed in any way or used commercially without permission from the journal.
Key Words: therapeutic drug monitoring, thiopurines, azathioprine, mercaptopurine, 6-thioguanine nucleotides, 6-methylmercaptopurine

(Ther Drug Monit 2017;39:584-588)

\section{INTRODUCTION}

Thiopurines, available as the derivatives azathioprine (AZA), mercaptopurine (MP), and thioguanine (TG), are antimetabolite and immunosuppressive drugs, developed over 65 years ago, initially for the treatment of acute lymphoblastic leukemia. ${ }^{1}$ Subsequently, thiopurines were slowly adapted for the prevention of organ transplant rejection and the management of chronic inflammatory diseases, including rheumatoid arthritis and inflammatory bowel disease (IBD). ${ }^{2-4}$ Currently, AZA and MP have proven to be effective as a monotherapy in maintaining steroid-free remission in both Crohn disease and ulcerative colitis. ${ }^{5,6}$ Thiopurines may also be used in combination with anti-tumor necrosis factor agents in IBD to optimize therapeutic efficacy and reduce secondary loss of response. ${ }^{7,8}$ Moreover, TG, a nonconventional thiopurine derivative, is considered as an escape drug for patients with IBD who failed AZA or MP because of inefficacy, intolerance, or toxicity. ${ }^{9,10}$

Strategies to optimize thiopurine therapy have demonstrated to be valuable in the management of IBD. ${ }^{11}$ Currently, therapeutic drug monitoring (TDM) of thiopurine metabolites may be used to increase clinical efficacy and reduce drugassociated toxicity. ${ }^{12-15}$ In this procedure, 6-thioguanine nucleotides (6-TGN) and 6-methylmercaptopurine (6-MMP) metabolites are measured and related to therapeutic response and adverse events, respectively. There remains a controversy on the additional value of TDM of thiopurines in optimizing IBD treatment because several studies addressed conflicting results on the association between thiopurine metabolite levels and clinical outcome in IBD. ${ }^{15-20}$ Nevertheless, the use of TDM of thiopurines in patients with IBD, either as a routine or in specific therapy-associated circumstances, is increasingly being applied in the daily clinical practice. When TDM is applied, the interpretation of measured metabolite levels and translation into clinical outcome should be done carefully, partially as a result of several analytical obstacles in this procedure. ${ }^{16,21}$ These limitations could create general misconceptions regarding TDM of thiopurines and impair its utilization. This review is intended to describe the analytical pitfalls of TDM of thiopurines and to provide suggestions to improve TDM utilization in daily practice to optimize thiopurine therapy in IBD. 


\section{DRUG METABOLISM}

Thiopurines interact in cell processes involved in inflammation and proliferation and require bioactivation through an extensive metabolism with involvement of multiple enzymes. ${ }^{22}$ Thiopurine metabolism occurs intracellularly and is determined by cell-specific characteristics and activity of genetically influenced enzymes. Hence, thiopurines operate effectively in target cells, such as leukocytes, but their drug metabolism is interindividually highly variable. Thioguanine, AZA, and MP are all converted into pharmacologically active 6-TGN, which consist of 6-thioguanine monophosphate (6-TGMP), 6-thioguanine diphosphate (6-TGDP), and 6-thioguanine triphosphate (6TGTP) (Fig. 1). ${ }^{15}$ In early studies, performed in patients with leukemia treated with high-dosage of thiopurines, the mechanism of action was ascribed to the incorporation of fraudulent 6-TGN into DNA, inhibiting cell proliferation. ${ }^{23}$ In this study, it is assumed that the 6-TGTP nucleotides, in particular, contribute to immunosuppressive effects in the treatment of IBD by binding Ras-related C3 botulinum toxin substrate 1 (Rac1) and subsequently inducing T-cell apoptosis. ${ }^{24,25}$

Azathioprine is directly converted into 6-MP, which is metabolized into different metabolites depending on the type of pathway. For 6-MP, 6-TGN are formed by the purine salvage pathway, requiring the enzymatic activity of hypoxanthine-guanine phosphoribosyl transferase, inosine monophosphate dehydrogenase (IMPDH), and guanosine monophosphate synthetase, respectively. Furthermore, 6-MP may also be converted into 6-thiouric acid (6-TUA) by xanthine oxidase or into potentially toxic 6-MMP metabolites and 6-methylmercaptopurine ribonucleotides (6-MMPR) by thiopurine S-methyltransferase (TPMT). Thioguanine is converted into 6-TGN, through the purine salvage pathway in 1 step and without the formation of the toxic 6-MMP or 6MMPR metabolites. ${ }^{26}$ Thioguanine may also be metabolized into 6-TUA or 6-methylthioguanine (6-MTG) through competing pathways.

\section{TDM OF THIOPURINES IN IBD}

The definite role of routine measurements of 6-TGN and 6-MMP levels in the management of IBD has not been well established yet. Current data on this issue are derived from (mostly) small-sized and poorly conducted studies as addressed in 2 meta-analyses. ${ }^{27,28}$ In both studies, a positive correlation between clinical remission and high 6-TGN levels was demonstrated, and diversity in the assays applied for TDM and the instruments used to determine the disease activity were reported as possible explanations for the conflicting results among the studies. ${ }^{27,28}$ Using multilevel analysis, Nguyen et al also observed positive correlations between weight-based AZA dosages and 6-TGN levels, and 6-MMP levels and the 6MMP/6TGN ratio, in pediatric patients with IBD treated with AZA. ${ }^{29}$

Reference values for therapeutic response and toxicity of thiopurine metabolites are method specific and constituted by the association of clinical outcome with measured levels in a disease with a varying activity course by nature. Numerous high-performance liquid chromatography methods to determine thiopurine metabolites are described, of which the methods by Lennard (1987) and Dervieux (1998) are the most commonly used methods. ${ }^{30-33}$ Both were originally developed for patients with leukemia treated with high dosages of thiopurines as chemotherapeutic agents. Because successful thiopurine treatment led to the disappearance of leukocytes in patients with leukemia, 6-TGN and 6-MMP levels were consequently measured in erythrocytes. ${ }^{1}$ In the method developed by Dervieux, erythrocyte 6-TGN concentration is 2.6-fold higher compared with the Lennard method because of a higher hydrolysis of TG phosphate groups. ${ }^{34}$ Therefore, it is important to take note of the applied method. In both methods, 6-MMP levels are similar. Both 6-TGN and 6-MMP concentrations are expressed as pmol/ $8 \times 10^{8} \mathrm{red}$ blood cells (RBCs).

In AZA or MP therapy, it is considered that erythrocyte 6-TGN levels between 230 and $450 \mathrm{pmol} / 8 \times 10^{8} \mathrm{RBC}$ are

FIGURE 1. Simplified metabolic pathway of thiopurines. Bold lines represent the purine salvage pathway in which the pharmacologically active metabolites [6-thioguanine nucleotides (6-TGN)] are formed, whereas dotted lines represent the competing pathways. Azathioprine (AZA) is converted into mercaptopurine (MP) by separating the imidazole group. Mercaptopurine is subsequently metabolized into 6TGN through a multistep pathway, by the enzymes hypoxanthineguanine phosphoribosyl transferase (HGPRT), inosine monophosphate

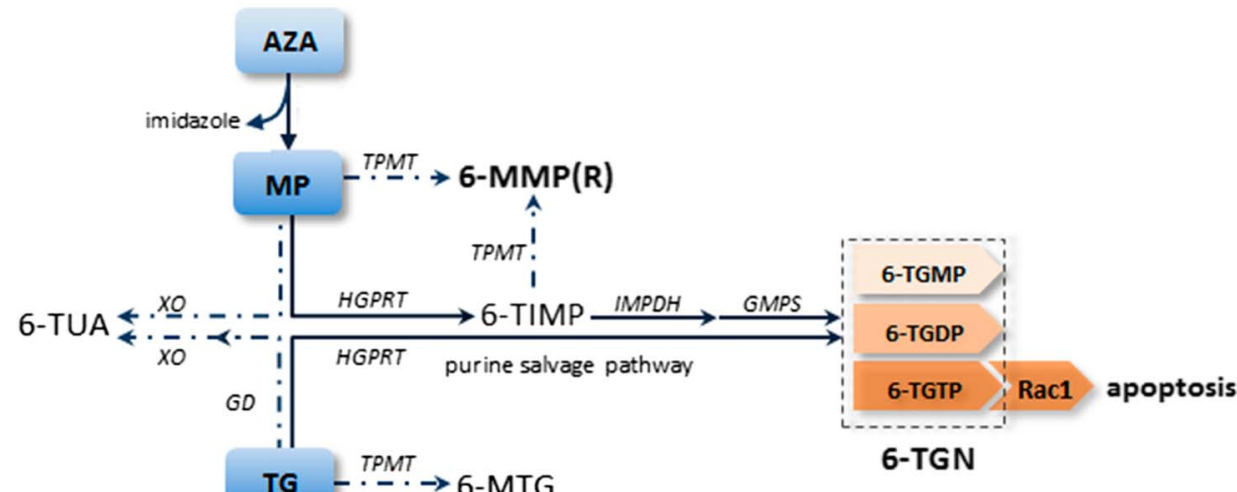
dehydrogenase (IMPDH), and guanosine monophosphate synthetase (GMPS). Through competing pathways, MP is converted by xanthine oxidase (XO) into 6-thiouric acid (6-TUA) or by TPMT into 6-methylmercaptopurine (6-MMP) and 6-methylmercaptopurine ribonucleotides (6-MMPR). Thioguanine (TG) is converted into 6-TGN in 1 step through the purine salvage pathway for which only HGPRT is necessary. Thioguanine may also be transformed into 6-methylthioguanine (6-MTG) by TPMT or into 6-TUA by guanine deaminase (GD) and XO. 6-TGN consists of 6-thioguanine monophosphate (6-TGMP), 6-thioguanine diphosphate (6-TGDP), and 6-thioguanine triphosphate (6-TGTP). The 6-TGTP nucleotides target Rac1 and finally induce T-cell apoptosis. 
associated with a higher probability of therapeutic response (Fig. 2). ${ }^{12-14,28}$ On the contrary, 6-TGN levels below 230 and above $450 \mathrm{pmol} / 8 \times 10^{8} \mathrm{RBC}$ are related to ineffectiveness and myelotoxicity, respectively. ${ }^{12,15,21,22,26}$ Furthermore, 6MMP levels above $5700 \mathrm{pmol} / 8 \times 10^{8} \mathrm{RBC}$ increase the risk of hepatotoxicity and myelotoxicity, probably due to the inhibition of de novo purine synthesis. ${ }^{35}$ If both 6-TGN and 6MMP levels are (nearly) undetectable, noncompliance should be considered. ${ }^{36}$ In TG therapy, erythrocyte 6-TGN levels are generally much higher, whereas leukocyte 6-TGN levels are similar to or even lower than in AZA and MP therapy. ${ }^{37}$ In studies on TG therapy in patients with IBD, 6-TGN levels were not related to the therapeutic response or toxicity. ${ }^{38,39} \mathrm{In}$ addition, even TG in low dose was converted into relatively high 6-TGN levels, without inducing myelotoxicity or affecting hematological parameters. ${ }^{38}$

\section{PITFALLS OF TDM OF THIOPURINES IN IBD}

In daily clinical practice, measuring thiopurine metabolites might be relevant after initiating therapy, to monitor compliance with therapy, after dose adjustments and at the time of adverse events or clinical relapse. ${ }^{40}$ Interpretation of these metabolite levels and their association with therapeutic response or toxicity is challenging, partially as a consequence of analytical limitations of these assays. Knowledge of these limitations is essential for the optimal utilization of thiopurine drug monitoring in IBD.

\section{Biochemical Limitations}

The metabolism of thiopurines requires nucleated cells, such as leukocytes, which are assumed to be the main target

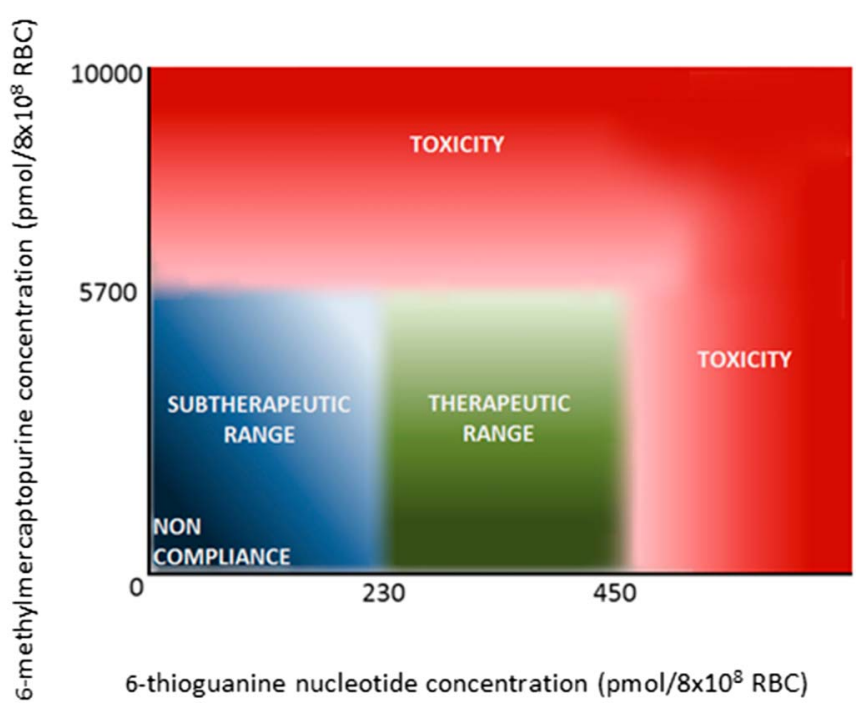

FIGURE 2. Therapeutic and toxicity reference ranges in therapeutic drug monitoring of thiopurines in IBD. The association of erythrocyte 6-thioguanine nucleotides ( $x$-axis) and erythrocyte 6-methylmercaptopurine ( $y$-axis) metabolites with therapeutic response and toxicity to azathioprine and mercaptopurine treatment in IBD. Reference values are depicted in the method by Lennard. ${ }^{33} \mathrm{RBC}$, red blood cell. cells of these drugs. ${ }^{15}$ In TDM, however, thiopurine metabolites are determined in erythrocytes and are used as a surrogate for intracellular metabolite levels in target cells. This method derives from the original indication of thiopurines in patients with leukemia, in whom leukocytes were not available during a successful induction cancer treatment. Interestingly, erythrocytes and leukocytes have different (cell-specific) characteristics and therefore an incomparable thiopurine metabolism. The most important difference is that leukocytes are nucleated cells, whereas (mature) erythrocytes have no nucleus and a smaller cell volume. In addition, erythrocytes lack functional IMPDH, an important key enzyme, essential in the purine salvage pathway of AZA and MP to convert into 6-TGN. ${ }^{16}$ Therefore, the measured 6-TGN in erythrocytes must have been absorbed from tissues that are able to convert thiopurines into 6-TGN. Consequently, surrogate erythrocyte 6-TGN reflect exposure in other (mainly hepatic) tissues more than in the target cells. ${ }^{16}$ Hence, erythrocyte 6-TGN do not directly reflect the pharmacodynamics of thiopurines, whereas the assessment of 6-TGN in leukocytes seems more appropriate. In several studies, most of which were performed in children with acute lymphoblastic leukemia, erythrocytary 6-TGN levels appeared to be correlated to leukocytary 6-TGN levels. ${ }^{12,37,41-43}$

Interestingly, TG does not need IMPDH for conversion into 6-TGN, which may be one of the underlying reasons for the substantially higher erythrocyte 6-TGN levels during TG therapy (Fig. 1). ${ }^{38}$ In leukocytes, however, MP and TG administered in standard dosages led to comparable 6-TGN levels. ${ }^{37}$

Furthermore, other factors, such as the inconsistent bioavailability of thiopurines and concomitant drug use (eg, allopurinol and 5-aminosalicylic acid [5-ASA]) may influence drug metabolism and consequently the drug monitoring of thiopurines as well. ${ }^{21}$

\section{Methodological Limitations}

Currently, methodological assays used for determining thiopurine metabolite levels have several limitations, resulting in an impaired validity of these tests. A weakness of these methods derives from the indirect assessment of 6-TGN as hydrolysis products, without distinguishing between nucleotides and unwanted residual products (ie, ribosides and deoxynucleotides). ${ }^{44}$ Moreover, these methods fail to identify separate monophosphate, diphospahte, and triphosphate nucleotides. ${ }^{44}$

Another pitfall of TDM of thiopurines is the limited stability of erythrocyte 6-TGN levels, which depends on the time and storage conditions. ${ }^{45}$ Blood for thiopurine metabolite measurements may be stored for 48 hours at room temperatures and for up to $3-4$ days at $4-8^{\circ} \mathrm{C} .^{30,33,45} \mathrm{~A}$ minimal volume of $0.5 \mathrm{~mL}$ of blood should be drawn in EDTA or heparin-sprayed tubes to prevent clotting. A generalized and standardized laboratory practice regarding sample collection, storage, and shipment conditions in thiopurine metabolite assessment is usually absent. ${ }^{16}$ Therefore, it must be taken into account that analyzed 6-TGN levels differ between different laboratories. This is essential when thiopurine metabolite analyses are not performed in the own laboratory and blood samples have to be transferred elsewhere. As a consequence of the erythrocyte 6-TGN instability over time, measured metabolites may be lower in such cases. 


\section{Novel Targets}

Because current TDM of thiopurines is hampered by biochemical and methodological limitations, improved assays and specific markers are warranted. Regarding the specific immunosuppressive effects of Rac1 binding of 6-TGTP, multiple methods have been developed to measure 6-TGMP, 6-TGDP, and 6-TGTP levels separately. ${ }^{44,46,47}$ Early data suggested that high levels of 6-TGTP together with low levels of 6-TGDP were related to therapeutic response. ${ }^{46,47}$ Furthermore, Rac1 itself was proposed as a potential early marker of clinical outcome, in which decreased expression of Rac1 expression was associated with drug efficacy. ${ }^{48}$ In addition, several hematologic parameters, including leukocyte count, platelet count, and the change of mean corpuscular volume, were assessed in multiple studies with conflicting results. ${ }^{49-51}$ Currently, these methods are not yet applicable in routine clinical practice and require further research.

\section{CONCLUSION}

Monitoring metabolite levels may be useful for guiding thiopurine treatment in patients with IBD. However, a definite place for TDM of thiopurines in the management of IBD is challenging, partially as a consequence of analytical hurdles in this procedure. The metabolism of thiopurines is complex and varies extensively between individuals, mainly due to the involvement of various activities of enzymes, which is at least partially due to genetic variation. Likewise, thiopurine metabolism is cell specific and requires particular enzymes and nucleated cells for 6-TGN formation. In the current TDM, metabolite levels are assessed in erythrocytes, whereas leukocytes are considered as the main target cells of these drugs. Differences in these cells may impair the reliability of translating metabolite levels to clinical outcome in TDM. Furthermore, currently used methods do not distinguish between nucleotides and their unwanted residual products during hydrolysis nor among individual monophosphate, diphosphate, and triphosphate nucleotides. Monitoring of individual 6-TGTP nucleotides, preferably in nonstimulated target cells, and definition of a standardized analytical procedure regarding the measurement of erythrocyte TGN and storage of samples could be a major advance in TDM of thiopurines in patients with IBD.

\section{REFERENCES}

1. Elion GB. The purine path to chemotherapy. Science. 1989;244:41-47.

2. Karran P, Attard N. Thiopurines in current medical practice: molecular mechanisms and contributions to therapy-related cancer. Nat Rev Cancer. 2008;8:24-36.

3. Derijks LJ, Gilissen LP, Hooymans PM, et al. Review article: thiopurines in inflammatory bowel disease. Aliment Pharmacol Ther. 2006;24: 715-729.

4. Zweiman B. Immunosuppressive effects of specific classes of agents with special reference to organ transplantation. Immunosuppression by thiopurines. Transpl Proc. 1973;5:1197-1201.

5. Mowat C, Cole A, Windsor A, et al. Guidelines for the management of inflammatory bowel disease in adults. Gut. 2011;60:571-607.

6. Ginsburg PM, Dassopoulos T. Steroid dependent ulcerative colitis: azathioprine use is finally "evidence-based". Inflamm Bowel Dis. 2006;12: 921-922.

7. Panaccione R, Ghosh S, Middleton S, et al. Combination therapy with infliximab and azathioprine is superior to monotherapy with either agent in ulcerative colitis. Gastroenterology. 2014;146:392-400.e393.
8. Colombel JF, Sandborn WJ, Reinisch W, et al. Infliximab, azathioprine, or combination therapy for Crohn's disease. $N$ Engl J Med. 2010;362: 1383-1395.

9. Derijks LJ, de Jong DJ, Gilissen LP, et al. 6-Thioguanine seems promising in azathioprine- or 6-mercaptopurine-intolerant inflammatory bowel disease patients: a short-term safety assessment. Eur J Gastroenterol Hepatol. 2003;15:63-67.

10. de Boer NK, Derijks LJ, Gilissen LP, et al. On tolerability and safety of a maintenance treatment with 6-thioguanine in azathioprine or 6mercaptopurine intolerant IBD patients. World J Gastroenterol. 2005; 11:5540-5544.

11. Meijer B, Seinen ML, van Egmond R, et al. Optimizing thiopurine therapy in inflammatory bowel disease among 2 real-life intercept cohorts: effect of allopurinol comedication? Inflamm Bowel Dis. 2017;23: 2011-2017.

12. Cuffari C, Seidman EG, Latour S, et al. Quantitation of 6-thioguanine in peripheral blood leukocyte DNA in Crohn's disease patients on maintenance 6-mercaptopurine therapy. Can J Physiol Pharmacol. 1996;74: 580-585.

13. Cuffari C, Hunt S, Bayless T. Utilisation of erythrocyte 6-thioguanine metabolite levels to optimise azathioprine therapy in patients with inflammatory bowel disease. Gut. 2001;48:642-646.

14. Chrzanowska M, Kolecki P, Duczmal-Cichocka B, et al. Metabolites of mercaptopurine in red blood cells: a relationship between 6-thioguanine nucleotides and 6-methylmercaptopurine metabolite concentrations in children with lymphoblastic leukemia. Eur J Pharm Sci. 1999;8:329-334.

15. Dubinsky MC, Lamothe S, Yang HY, et al. Pharmacogenomics and metabolite measurement for 6-mercaptopurine therapy in inflammatory bowel disease. Gastroenterology. 2000;118:705-713.

16. Duley JA, Florin TH. Thiopurine therapies: problems, complexities, and progress with monitoring thioguanine nucleotides. Ther Drug Monit. 2005;27:647-654.

17. Kwan LY, Devlin SM, Mirocha JM, et al. Thiopurine methyltransferase activity combined with 6-thioguanine metabolite levels predicts clinical response to thiopurines in patients with inflammatory bowel disease. Dig Liver Dis. 2008;40:425-432.

18. Gonzalez-Lama Y, Bermejo F, Lopez-Sanroman A, et al. Thiopurine methyl-transferase activity and azathioprine metabolite concentrations do not predict clinical outcome in thiopurine-treated inflammatory bowel disease patients. Aliment Pharmacol Ther. 2011;34:544-554.

19. Gilissen LP, Wong DR, Engels LG, et al. Therapeutic drug monitoring of thiopurine metabolites in adult thiopurine tolerant IBD patients on maintenance therapy. J Crohns Colitis. 2012;6:698-707.

20. Reinshagen M, Schutz E, Armstrong VW, et al. 6-thioguanine nucleotideadapted azathioprine therapy does not lead to higher remission rates than standard therapy in chronic active crohn disease: results from a randomized, controlled, open trial. Clin Chem. 2007;53:1306-1314.

21. Van Asseldonk DP, de Boer NK, Peters GJ, et al. On therapeutic drug monitoring of thiopurines in inflammatory bowel disease; pharmacology, pharmacogenomics, drug intolerance and clinical relevance. Curr Drug Metab. 2009;10:981-997.

22. Quemeneur L, Gerland LM, Flacher M, et al. Differential control of cell cycle, proliferation, and survival of primary $\mathrm{T}$ lymphocytes by purine and pyrimidine nucleotides. J Immunol. 2003;170:4986-4995.

23. Marathias VM, Sawicki MJ, Bolton PH. 6-Thioguanine alters the structure and stability of duplex DNA and inhibits quadruplex DNA formation. Nucleic Acids Res. 1999;27:2860-2867.

24. Seinen ML, van Nieuw Amerongen GP, de Boer NK, et al. Rac attack: modulation of the small gtpase rac in inflammatory bowel disease and thiopurine therapy. Mol Diagn Ther. 2016;20:551-557.

25. Tiede I, Fritz G, Strand S, et al. CD28-dependent Rac1 activation is the molecular target of azathioprine in primary human $\mathrm{CD} 4+\mathrm{T}$ lymphocytes. J Clin Invest. 2003;111:1133-1145.

26. de Boer NK, van Bodegraven AA, Jharap B, et al. Drug Insight: pharmacology and toxicity of thiopurine therapy in patients with IBD. Nat Clin Pract Gastroenterol Hepatol. 2007;4:686-694.

27. Moreau AC, Paul S, Del Tedesco E, et al. Association between 6thioguanine nucleotides levels and clinical remission in inflammatory disease: a meta-analysis. Inflamm Bowel Dis. 2014;20:464-471.

28. Osterman MT, Kundu R, Lichtenstein GR, et al. Association of 6thioguanine nucleotide levels and inflammatory bowel disease activity: a meta-analysis. Gastroenterology. 2006;130:1047-1053. 
29. Nguyen TV, Vu DH, Nguyen TM, et al. Relationship between azathioprine dosage and thiopurine metabolites in pediatric IBD patients: identification of covariables using multilevel analysis. Ther Drug Monit. 2013;35:251-257.

30. Lennard L. Assay of 6-thioinosinic acid and 6-thioguanine nucleotides, active metabolites of 6-mercaptopurine, in human red blood cells. $J$ Chromatogr. 1987;423:169-178.

31. Lennard L, Singleton HJ. High-performance liquid chromatographic assay of the methyl and nucleotide metabolites of 6-mercaptopurine: quantitation of red blood cell 6-thioguanine nucleotide, 6-thioinosinic acid and 6-methylmercaptopurine metabolites in a single sample. J Chromatogr. 1992;583:83-90.

32. Dervieux T, Boulieu R. Simultaneous determination of 6-thioguanine and methyl 6-mercaptopurine nucleotides of azathioprine in red blood cells by HPLC. Clin Chem. 1998;44:551-555.

33. Lennard L, Maddocks JL. Assay of 6-thioguanine nucleotide, a major metabolite of azathioprine, 6-mercaptopurine and 6-thioguanine, in human red blood cells. J Pharm Pharmacol. 1983;35:15-18.

34. Shipkova M, Armstrong VW, Wieland E, et al. Differences in nucleotide hydrolysis contribute to the differences between erythrocyte 6thioguanine nucleotide concentrations determined by two widely used methods. Clin Chem. 2003;49:260-268.

35. Dubinsky MC, Yang H, Hassard PV, et al. 6-MP metabolite profiles provide a biochemical explanation for 6-MP resistance in patients with inflammatory bowel disease. Gastroenterology. 2002;122:904-915.

36. Wong DR, Derijks LJ, den Dulk MO, et al. The role of xanthine oxidase in thiopurine metabolism: a case report. Ther Drug Monit. 2007;29:845-848.

37. Lancaster DL, Patel N, Lennard L, et al. Leucocyte versus erythrocyte thioguanine nucleotide concentrations in children taking thiopurines for acute lymphoblastic leukaemia. Cancer Chemother Pharmacol. 2002;50:33-36.

38. de Boer NK, Derijks LJ, Keizer-Garritsen JJ, et al. Extended thiopurine metabolite assessment during 6-thioguanine therapy for immunomodulation in Crohn's disease. J Clin Pharmacol. 2007;47:187-191.

39. de Boer NK, de Graaf P, Wilhelm AJ, et al. On the limitation of 6tioguaninenucleotide monitoring during tioguanine treatment. Aliment Pharmacol Ther. 2005;22:447-451.

40. Al Hadithy AF, de Boer NK, Derijks LJ, et al. Thiopurines in inflammatory bowel disease: pharmacogenetics, therapeutic drug monitoring and clinical recommendations. Dig Liver Dis. 2005;37:282-297.
41. Warren DJ, Andersen A, Slordal L. Quantitation of 6-thioguanine residues in peripheral blood leukocyte DNA obtained from patients receiving 6-mercaptopurine-based maintenance therapy. Cancer Res. 1995;55: 1670-1674

42. Dervieux T, Chu Y, Su Y, et al. HPLC determination of thiopurine nucleosides and nucleotides in vivo in lymphoblasts following mercaptopurine therapy. Clin Chem. 2002;48:61-68.

43. Erdmann GR, Steury JC, Carleton BC, et al. Reversed-phase highperformance liquid chromatographic approach to determine total lymphocyte concentrations of 6-thioguanine, methylmercaptopurine and methylthioguanine in humans. J Chromatogr. 1991;571:149-156.

44. Vikingsson S, Andersson D, Almer S, et al. Novel assay to improve therapeutic drug monitoring of thiopurines in inflammatory bowel disease. J Crohns Colitis. 2014;8:1702-1709.

45. de Graaf P, Vos RM, de Boer NH, et al. Limited stability of thiopurine metabolites in blood samples: relevant in research and clinical practise. $J$ Chromatogr B Analyt Technol Biomed Life Sci. 2010;878:1437-1442.

46. Neurath MF, Kiesslich R, Teichgraber U, et al. 6-thioguanosine diphosphate and triphosphate levels in red blood cells and response to azathioprine therapy in Crohn's disease. Clin Gastroenterol Hepatol. 2005;3: 1007-1014.

47. Karner S, Shi S, Fischer C, et al. Determination of 6-thioguanosine diphosphate and triphosphate and nucleoside diphosphate kinase activity in erythrocytes: novel targets for thiopurine therapy? Ther Drug Monit. 2010;32:119-128.

48. Seinen ML, van Nieuw Amerongen GP, de Boer NK, et al. Rac1 as a potential pharmacodynamic biomarker for thiopurine therapy in inflammatory bowel disease. Ther Drug Monit. 2016;38:621-627.

49. Dujardin RW, Meijer B, de Boer NK, et al. Usefulness of mean corpuscular volume as a surrogate marker for monitoring thiopurine treatment in inflammatory bowel disease. Eur J Gastroenterol Hepatol. 2016;28: 991-996.

50. Kopylov U, Battat R, Benmassaoud A, et al. Hematologic indices as surrogate markers for monitoring thiopurine therapy in IBD. Dig Dis Sci. 2015;60:478-484.

51. Heerasing NM, Ng JF, Dowling D. Does lymphopenia or macrocytosis reflect 6-thioguanine levels in patients with inflammatory bowel disease treated with azathioprine or 6-mercaptopurine? Intern Med J. 2016;46: 465-469. 